\title{
A two centre study of the dose-response relation for transscleral diode laser cyclophotocoagulation in refractory glaucoma
}

\author{
C C Murphy, C A M Burnett, P G D Spry, D C Broadway, J P Diamond
}

Br J Ophthalmol 2003;87:1252-1257

Background/aims: Transscleral diode laser cyclophotocoagulation ("cyclodiode") is widely used to treat refractory glaucoma. The main aims of this study were to investigate the dose-response relation of cyclodiode and to evaluate possible predictive factors that would help establish optimum treatment parameters.

Methods: A retrospective analysis of the case notes of 263 eyes of 238 consecutive patients who underwent transscleral diode laser cyclophotocoagulation at two centres was undertaken.

Results: Mean intraocular pressure (IOP) decreased significantly from $40.7 \mathrm{~mm} \mathrm{Hg}$ (SD 13.7) before cyclodiode therapy to $17.7 \mathrm{~mm} \mathrm{Hg}$ (SD 10.9) post-treatment, a reduction of $52.6 \%$ ( $p=0.0001$ ).

See end of article for authors' affiliations

....................

Correspondence to: Mr J P Diamond, Bristol Eye Hospital, Lower Maudlin Street, Bristol, BS1 2LX, UK; i.p.diamond@ bristol.ac.uk

Accepted for publication 6 January 2003 Following cyclodiode, $89 \%$ of patients achieved an IOP of less than $22 \mathrm{~mm} \mathrm{Hg}$ or a greater than $30 \%$ drop in IOP. Hypotony occurred in $9.5 \%$ of patients, $76 \%$ of whom had neovascular glaucoma. A linear dose relation response was found for the 122 eyes with neovascular glaucoma $(p=0.001)$ but not for the group as a whole. Treatment failure was associated with male sex (multivariate regression analysis, $p=0.008$ ) and low mean energy per treatment session (univariate analysis alone, $p=0.016$ ). High pretreatment IOP $(p=0.031)$ and high mean energy per treatment episode $(p=0.001)$ appeared to be associated with the occurrence of hypotony, although multivariate analysis did not support this finding.

Conclusion: Cyclodiode therapy is highly effective but there is a significant risk of hypotony, which may be reduced by applying lower energy in cases of very high pretreatment IOP and in neovascular glaucoma. The dose-response association remains unpredictable, although a linear relation was found for neovascular glaucoma.
T ransscleral diode laser cyclophotocoagulation (cyclodiode) has been established as a relatively safe and effective intervention for glaucoma resistant to conventional management. ${ }^{1-7}$ The procedure has become increasingly popular in recent years as an alternative to the surgical options for refractory glaucoma, such as antimetabolite augmented trabeculectomy and tube shunt surgery. It has been shown to be safer than other cyclodestructive procedures, such as Nd:YAG laser cyclophotocoagulation and cyclocryotherapy, which present a significant risk of hypotony and phthisis because of excessive ciliary body ablation. ${ }^{8-10}$ However, theoutcomeofcyclodiodetherapyisunpredictableand multiple treatments may be required to achieve the desired result. ${ }^{11}{ }^{12}$ Furthermore, no consensus exists for an optimum treatment protocol.

The main objectives of this study were to (a) evaluate the relation between total diode laser energy delivered and the change in intraocular pressure (IOP), and (b) identify possible predictive factors that could help establish optimum treatment parameters. Secondary goals were to study the efficacy and safety of diode laser cyclophotocoagulation and to evaluate the IOP lowering effect following each treatment session in patients receiving multiple treatments to help with management planning and patient counselling.

\section{METHODS}

A retrospective analysis was carried out using the case notes of patients who had undergone cyclodiode therapy in two ophthalmic centres in the United Kingdom, the Bristol Eye Hospital and the Norfolk and Norwich University Hospital. For each centre, the case notes of consecutive patients undergoing treatment over a 30 month period were reviewed.
Data were collected pertaining to patient demographics, glaucoma diagnosis, and cyclodiode laser delivery. In addition, the following data were recorded before and after cyclodiode therapy: IOP, antiglaucoma medications (including oral acetazolamide), visual acuity, and complications.

Indications for cyclodiode therapy included (1) inadequate IOP control and advanced glaucoma in the presence of maximum tolerated medical therapy in patients who had had filtration surgery in the past, or for whom filtration surgery was declined or deemed to present too high a risk of failure, and (2) severe ocular hypertension causing pain and/or corneal oedema despite maximum medical antiglaucoma therapy.

Transscleral diode laser cyclophotocoagulation was performed in each centre using the OcuLight SLx $810 \mathrm{~nm}$ diode laser photocoagulator and the Iris G-probe, both from Iris Medical Instruments (Mountain View, CA, USA). Treatments were administered under local anaesthesia ( $2 \%$ lignocaine given as a peribulbar or retrobulbar injection) or general anaesthesia. Transillumination was utilised in the majority of cases and in particular when the location of the ciliary body was uncertain-for example, congenital glaucoma. The Gprobe was placed approximately $1.2 \mathrm{~mm}$ posterior to the limbus with an axial orientation. There was no set treatment protocol. In most cases, laser was applied over 270 degrees of the ciliary body, excluding the superonasal quadrant or in all four quadrants with sparing of the 3 and 9 o'clock regions (site of the long ciliary nerves and vessels). The number of burns and the energy per burn were not standardised, but varied from case to case. In general, higher total energy was applied in refractory cases, eyes with very high IOP, eyes which were already blind or those where the risk of hypotony 
was deemed to be low. Conversely, lower total energy was applied for eyes with marginal pressure elevation, "sighted eyes," "only eyes," or other cases where the risk of hypotony was considered to be high. In this latter group it was usually felt that the risk of repeated laser treatment (that is, undertreatment) was less significant than the risk of hypotony.

Postoperatively, the treated eye was patched for 1-6 hours and topical steroid drops (usually dexamethasone $1 \%$ ) were applied four times daily and tapered as any inflammation subsided. Antiglaucoma medications were reduced and discontinued according to the IOP response. Patients were assessed within 2 weeks and at intervals of 1-6 months thereafter. At each clinic visit, visual acuity, IOP, and slit lamp findings including complications of cyclodiode therapy were recorded. Cyclodiode therapy was repeated if the IOP response was inadequate after a minimal follow up period of 1 month.

The IOP at the final follow up appointment was used to evaluate the relation between the total diode laser energy delivered and the drop in IOP. The treatment was deemed to be a success if the IOP at 6 months was between 5 and $21 \mathrm{~mm} \mathrm{Hg}$ or if there was a greater than $30 \%$ reduction in IOP, with or without topical antiglaucoma medications. Hypotony was defined as an IOP of less than $5 \mathrm{~mm} \mathrm{Hg}$ at the final follow up visit. The total cyclodiode energy (joules) was calculated by multiplying the number of laser burns by the duration (seconds) of each burn by the power (watts) of each burn. If the patient had more than one cyclodiode laser treatment, the total energies for each episode were added to give the overall total energy delivered. The method of data collection was identical in both centres.

The outcome of cyclodiode therapy was defined in terms of success rate, response rate, complication rate, and a cyclodiode efficacy index. The success rate was defined as the percentage of eyes achieving an IOP of between 5 and $21 \mathrm{~mm} \mathrm{Hg}$ or a greater than $30 \%$ drop in IOP following cyclodiode therapy at their final follow up visit. The response rate was defined as the percentage of patients achieving an IOP of less that $22 \mathrm{~mm} \mathrm{Hg}$ or a greater than $30 \%$ drop in IOP and included eyes that developed hypotony (IOP $<5 \mathrm{~mm} \mathrm{Hg}$ ). The cyclodiode efficacy index defined the sensitivity of the IOP to cyclodiode therapy and was determined as the ratio of the response rate (expressed as a decimal) to the mean number of treatment sessions. ${ }^{13}$ Thus, a cyclodiode efficacy index of 1.0 would indicate that all the eyes in an evaluated group achieved a satisfactory IOP response following a single treatment episode.

Linear regression analysis (least squares) was used to evaluate the dose-response relation between the total energy delivered during a course of cyclodiode therapy and the percentage drop or absolute drop in IOP. Multivariate logistic regression analysis was used to identify potential predictors for failure of cyclodiode therapy or the development of hypotony. The decrease in IOP and reduction in usage of antiglaucoma medications following cyclodiode therapy was evaluated using the Mann-Whitney test. Data analysis was performed using Stata 5.0 (Stata Corp, College station, TX, USA) and Graph Pad Prism 3.0 (GraphPad Software, San Diego, CA, USA).

\section{RESULTS}

A total of 263 eyes of 238 patients were evaluated with a mean follow up of 17 months (range 6-46); 136 eyes of 121 patients were treated at the Bristol Eye Hospital and 127 eyes of 117 patients at the Norfolk and Norwich University Hospital. In all, 123 patients $(52 \%)$ were female and 115 $(48 \%)$ patients were male. The mean age was 66 years (range 4-99). For the whole group, mean IOP decreased from

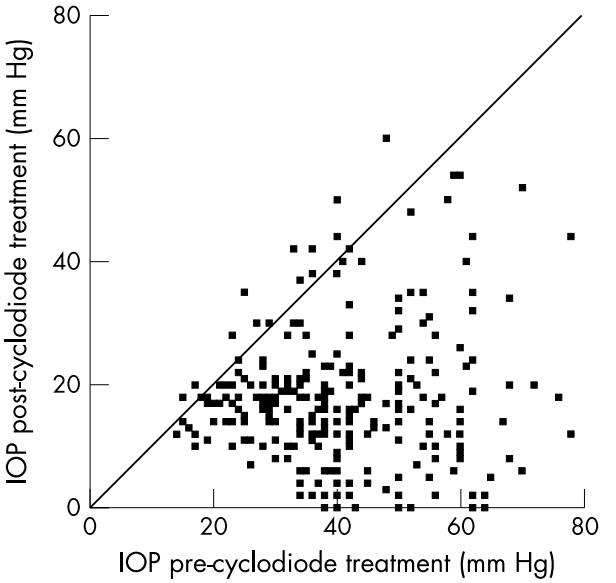

Figure 1 Scatter plot showing the change in IOP following cyclodiode therapy. All points below the diagonal line represent eyes in which there was a fall in IOP.

$40.7 \mathrm{~mm} \mathrm{Hg}$ (SD 13.7) before cyclodiode therapy to $17.7 \mathrm{~mm} \mathrm{Hg}$ (SD 10.9) post-treatment, a significant reduction of $52.6 \%(p=0.0001$, Fig 1$)$. The mean number of antiglaucoma medications used, including oral acetazolamide and topical treatments, before and after cyclodiode therapy was 1.7 (SD 1.2) and 0.8 (SD 1.0), respectively, the difference being highly significant $(p=0.0001)$. The mean total energy delivered was $155.2 \mathrm{~J}$ (SD 106.7) and the mean number of cyclodiode treatment sessions was 1.5 (SD 0.9, range 1-7).

Neovascular glaucoma accounted for $46.4 \%$ of the treated eyes, and was the result of central retinal vein occlusion (64.8\% of neovascular glaucoma eyes), diabetic retinopathy $(21.3 \%)$, ocular ischaemic syndrome $(4.1 \%)$, retinal detachment $(3.3 \%)$, or other causes $(6.6 \%)$. Other major diagnostic categories requiring cyclodiode therapy included primary open angle glaucoma (POAG, 12.9\%), uveitic glaucoma $(6.1 \%)$, aphakic glaucoma $(5.7 \%)$, chronic angle closure glaucoma (CACG, $5.7 \%$ ), and a group of various secondary glaucomas $(6.8 \%)$.

Data relating to the number of cyclodiode treatment sessions, effect on IOP, and the amount of laser energy applied for each diagnostic category are shown in Table 1. The IOP lowering effect was statistically significant for all diagnostic groups $(p<0.0001$, Mann-Whitney test $)$. The mean total energy delivered $(333 \mathrm{~J})$, mean energy per treatment session $(119 \mathrm{~J})$, and mean number of treatment sessions (3.5) were notably higher for congenital glaucoma than for other diagnostic categories.

The outcome of cyclodiode therapy for each diagnostic category is shown in Table 2. Of all categories, the best outcome occurred in eyes with chronic angle closure glaucoma, which had the highest success rate $(93 \%)$, the lowest re-treatment (13\%) and hypotony rates $(0 \%)$, and the highest cyclodiode efficacy index (0.84). Satisfactory outcomes also occurred in eyes with POAG, traumatic glaucoma, and glaucoma following keratoplasty. The lowest success rate $(75 \%)$ and cyclodiode efficacy index (0.21) occurred in congenital glaucoma.

The overall complication rate of cyclodiode therapy in this series was $12.2 \%$. Complications included hypotony, phthisis, persistent uveitis, hyphaema, and vitreous haemorrhage (Tables 3 and 4). Hypotony (IOP $<5 \mathrm{~mm} \mathrm{Hg}$ ), which occurred in $9.5 \%$ of all eyes, was seen in a variety of diagnostic groups, including neovascular, uveitic and aphakic glaucoma, and glaucoma secondary to vitreoretinal surgery. Of these, the maximum hypotony rate occurred in uveitic glaucoma (three of 16 eyes, $18.8 \%$ ). No complications occurred in the majority 
Table 1 IOP lowering effect and dose of cyclodiode laser applied for each diagnostic category

\begin{tabular}{|c|c|c|c|c|c|c|c|}
\hline $\begin{array}{l}\text { Diagnostic } \\
\text { category }\end{array}$ & $\begin{array}{l}\text { No of eyes } \\
\text { (\% total) }\end{array}$ & $\begin{array}{l}\text { Mean No of } \\
\text { treatments } \\
\text { (SD) }\end{array}$ & $\begin{array}{l}\text { Mean pre-laser } \\
\text { IOP (mm Hg) } \\
\text { (SD) }\end{array}$ & $\begin{array}{l}\text { Mean post-laser } \\
\text { IOP }(\mathrm{mm} \mathrm{Hg}) \\
\text { (SD) }\end{array}$ & $\begin{array}{l}\text { Mean percentage } \\
\text { change in } \\
\text { IOP (SD) }\end{array}$ & $\begin{array}{l}\text { Mean total } \\
\text { energy }(J)\end{array}$ & $\begin{array}{l}\text { Mean energy per } \\
\text { treatment episode } \\
\text { (J) }\end{array}$ \\
\hline All eyes & $263(100)$ & $1.5(0.9)$ & 40.7 (13.7) & $17.7(10.9)$ & $52.6(28)$ & $155.2(106.7)$ & $104.1(37.5)$ \\
\hline Neovascular & $122(46.4)$ & $1.4(0.6)$ & $48.3(12.1)$ & 17.4 (13.2) & $63.4(27.3)$ & $145.2(70.0)$ & $110.7(40.0)$ \\
\hline POAG & $34(12.9)$ & $1.6(0.9)$ & 30.6 (12.0) & $19.3(7.8)$ & $34.3(19.6)$ & 158.0 (128.3) & $93.0(32.5)$ \\
\hline Uveitic & $16(6.1)$ & $1.4(0.6)$ & $36.6(11.7)$ & $13.5(9.3)$ & 58.5 (30.5) & $128.6(61.7)$ & $89.4(28.8)$ \\
\hline Aphakic & $15(5.7)$ & $1.8(0.9)$ & $31.8(10.8)$ & $16.3(6.8)$ & $43.2(26.0)$ & 178.5 (113.4) & $90.4(40.7)$ \\
\hline CACG & $15(5.7)$ & $1.1(0.4)$ & $41.3(12.0)$ & $21.0(9.9)$ & 47.0 (23.4) & 108.7 (46.6) & 96.7 (35.8) \\
\hline Post-VR surgery & $14(5.3)$ & $1.1(0.4)$ & 32.6 (12.9) & $14.3(9.4)$ & $47.3(35.0)$ & $120.6(61.2)$ & $105.0(31.0)$ \\
\hline Traumatic & $11(4.2)$ & $1.8(0.8)$ & 38.0 (12.4) & $22.9(11.6)$ & $40.2(22.0)$ & 168.8 (110.7) & $93.6(24.6)$ \\
\hline Post-keratoplasty & $10(3.8)$ & $2.0(1.9)$ & $37.3(11.1)$ & $18.8(5.1)$ & 45.5 (21.6) & 225.6 (276.5) & $99.4(27.0)$ \\
\hline Congenital & $8(3.0)$ & $3.5(2.6)$ & $35.3(5.1)$ & $22.0(9.7)$ & 39.4 (23.2) & 332.8 (173.0) & $119.0(66.6)$ \\
\hline
\end{tabular}

The total cyclodiode energy $(\mathrm{J})$ was calculated by multiplying the number of laser burns by the duration (s) of each burn by the power (W) of each burn. If more than one laser treatment was applied, the total energies for each episode were added to give the overall total energy delivered. The mean energy per treatment episode was the total energy divided by the number of treatment episodes.

of the other diagnostic categories, while the two cases of hyphaema and vitreous haemorrhage resolved spontaneously. Persistent uveitis occurred in two of 122 eyes with rubeotic glaucoma (1.6\%), two of 16 eyes with uveitic glaucoma $(12.5 \%)$, and one of 34 eyes with POAG $(2.9 \%)$.

Visual acuity remained the same in $74.6 \%$ of the patients following cyclodiode therapy, decreased by more than one line in $19.8 \%$, and improved by more than one line in $5.3 \%$. All eyes that developed hypotony had a pre-cyclodiode visual acuity of hand movements or worse, with a median value of light perception.

The dose-response relation between the total energy delivered during the course of cyclodiode therapy and the percentage change in IOP is shown in Figure 2. There was no identified association between the total energy delivered and either the percentage change or absolute change in IOP for the group as a whole using linear regression analysis. However, analysis of the dose-response relation by diagnostic category revealed a linear relation for eyes with neovascular glaucoma $(\mathrm{p}=0.001)$.

Univariate and multivariate models of logistic regression analysis were used to identify possible predictors for failure of cyclodiode therapy. For this purpose, failure of cyclodiode therapy was defined as post-treatment IOP greater than $21 \mathrm{~mm} \mathrm{Hg}$ and a less than $30 \%$ reduction in IOP. The following variables were studied as potential factors predictive for treatment failure: age, sex, pretreatment IOP, total cyclodiode energy delivered, mean cyclodiode energy per treatment episode, the number of treatment episodes, and the mean time between treatments. For the group as a whole, male sex was associated with failure by both univariate and multivariate analyses $(p=0.008)$. Although the mean energy per treatment session was associated with failure on univariate analysis $(p=0.016)$, this finding was not supported by multivariate analysis.

These same predictive factors, together with poor visual acuity (defined as hand movements or worse) and glaucoma aetiology, were investigated using this methodology to identify factors associated with the development of hypotony, both for the group as a whole and separately for neovascular glaucoma. High pretreatment IOP $(p=0.031)$ and high mean energy per treatment episode $(p=0.001)$ were associated with hypotony for the group as a whole using univariate analysis, although multivariate analysis did not support this finding. For neovascular glaucoma alone, high mean energy per treatment episode appeared to be associated with hypotony $(p=0.005)$ on univariate analysis, although the multivariate modelling did not maintain this finding. Neither the aetiology of the neovascular glaucoma nor the initial visual acuity appeared to be associated with the development of post-cyclodiode hypotony. For further analysis, the neovascular glaucoma group was divided according to relatively good (counting fingers or better) or poor (hand movements or worse) visual acuity before cyclodiode therapy. The mean pre-treatment IOP was $41.5 \mathrm{~mm} \mathrm{Hg}$ in the good visual acuity group compared with $50.0 \mathrm{~mm} \mathrm{Hg}$ in the poor visual acuity group, a difference that was statistically significant $(p=0.0017)$. All of the cases of hypotony occurred in the poor visual acuity group and there was no difference in the mean energy per treatment between these groups.

A maximum of seven treatments were applied, with over $90 \%$ of eyes undergoing either one or two treatment sessions

Table 2 Clinical outcome of cyclodiode therapy for each diagnostic category

\begin{tabular}{lllllll}
\hline Diagnosis & $\begin{array}{l}\text { No of eyes } \\
(\% \text { total) }\end{array}$ & $\begin{array}{l}\text { Response rate } \\
(\%)\end{array}$ & $\begin{array}{l}\text { Success rate } \\
(\%)\end{array}$ & $\begin{array}{l}\text { Re-treatment } \\
\text { rate }(\%)\end{array}$ & $\begin{array}{l}\text { Hypotony rate Cyclodiode } \\
(\%)\end{array}$ \\
\hline effficacy index
\end{tabular}

The success rate was defined as the percentage of eyes achieving an IOP of between 5 and $21 \mathrm{~mm} \mathrm{Hg}$ or a greater than $30 \%$ reduction in IOP following cyclodiode therapy at their final follow up visit. The response rate was defined as the percentage of patients achieving an IOP of less that $22 \mathrm{~mm} \mathrm{Hg}$ or a greater than $30 \%$ reduction in IOP and included eyes that developed hypotony (IOP $<5 \mathrm{~mm} \mathrm{Hg}$ ). The cyclodiode efficacy index defined the sensitivity of the IOP to cyclodiode therapy and was calculated as the ratio of the response rate expressed as a decimal to the mean number of treatment sessions. 
Table 3 Complications of cyclodiode therapy

\begin{tabular}{lrc}
\hline Complications & No & $\%$ \\
\hline Hypotony (IOP $<5 \mathrm{~mm} \mathrm{Hg})$ & 25 & 9.5 \\
Phthisis & 14 & 5.3 \\
Uveitis & 5 & 1.9 \\
Hyphaema & 1 & 0.4 \\
Vitreous haemorrhage & 1 & 0.4 \\
\hline
\end{tabular}

(Table 5). The overall re-treatment rate was $34.2 \%$, being highest for traumatic glaucoma $(63.6 \%)$ and congenital glaucoma $(62.5 \%$, Table 2$)$. The mean time between treatments was 5.4 months (SD 5.4). Figure 3 shows the cumulative response rate following each additional cyclodiode treatment episode. $58.9 \%$ of eyes achieved a satisfactory IOP lowering from a single treatment episode, a figure that increased to $81.3 \%$ following the second treatment. Subsequent treatments did not increase the response rate significantly. However, the response rate for each treatment when considered alone remained in the region of $50 \%$ up to the fifth treatment episode, following which it decreased substantially to $20 \%$.

In comparing the results from the two centres no statistically significant differences were identified with respect to outcome or any of the factors investigated.

\section{DISCUSSION}

The results of the present study confirm the findings of other investigators that transscleral cyclodiode therapy is highly effective in lowering IOP. ${ }^{1-7}$ With the exception of congenital glaucoma, the response rate was in excess of $80 \%$ for all of the diagnostic categories evaluated in this study. Response rates over $90 \%$ were seen in POAG, chronic angle closure glaucoma, uveitic glaucoma, and glaucoma post-keratoplasty, while the response rate for the group as a whole was $89 \%$. A comparatively poor response rate of $75 \%$ for congenital glaucoma, as previously reported, ${ }^{14}$ confirmed that this form of glaucoma is relatively refractory to cyclodiode therapy, even when multiple treatments are applied. The difference between response and success rates in the present study reflects the incidence of hypotony, which was $9.5 \%$ for the group as a whole and occurred almost exclusively in eyes with neovascular glaucoma, uveitic glaucoma, or glaucoma post-vitreoretinal surgery.

The sensitivity to cyclophotocoagulation was measured using a cyclodiode efficacy index (0-1), in which a higher value indicated a greater sensitivity to treatment. Using this method, chronic angle closure glaucoma and glaucoma postvitreoretinal surgery were most sensitive to cyclophotocoagulation and primary congenital glaucoma, traumatic glaucoma and glaucoma post-keratoplasty were least sensitive. The re-treatment rate also reflected the sensitivity to cyclophotocoagulation and again, this was highest for congenital glaucoma, traumatic glaucoma, and glaucoma post-keratoplasty and lowest for chronic angle closure glaucoma.

The most serious adverse effects of cyclodiode therapy are hypotony and phthisis. In the present study, hypotony occurred in $25(9.5 \%)$ of 263 eyes and was common with uveitic glaucoma (18.8\%), neovascular glaucoma (15.6\%), and in glaucoma following vitreoretinal surgery (14.3\%). The hypotony rate reported in other studies has been highly variable, ranging from $0-18 \% .^{67121315}$ Neovascular glaucoma, which accounted for $46 \%$ of the eyes in this study, has previously been associated with a high risk of hypotony. ${ }^{2}$ Bloom and co-workers, however, reported a hypotony rate of only $1 \%$ in their series of 209 eyes that underwent cyclodiode,
Table 4 Hypotony and phthisis rate for each diagnostic category

\begin{tabular}{lll}
\hline Diagnosis & $\begin{array}{l}\text { Cases of hypotony } \\
\text { (\% total) }\end{array}$ & $\begin{array}{l}\text { Cases of phthisis } \\
\text { (\% total) }\end{array}$ \\
\hline All eyes & $25(9.5)$ & $14(5.3)$ \\
Neovascular & $19(15.6)$ & $10(8.2)$ \\
Uveitic & $3(18.80)$ & $2(12.5)$ \\
Post-VR surgery & $2(14.3)$ & $2(14.3)$ \\
Aphakic & $1(6.7)$ & 0 \\
CACG & 0 & 0 \\
POAG & 0 & 0 \\
Traumatic & 0 & 0 \\
Post-keratoplasty & 0 & 0 \\
Congenital & 0 & 0 \\
\hline
\end{tabular}
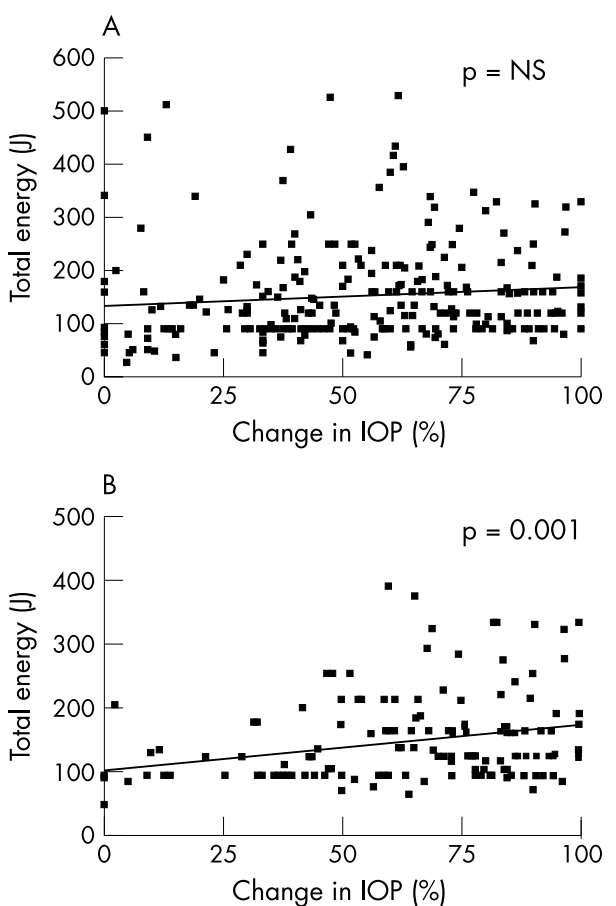

Figure 2 Dose-response for the whole group (A) and for neovascular glaucoma (B). In neovascular glaucoma there was a linear relation between the total energy applied and the percentage change in IOP following cyclodiode therapy $(p=0.001)$. NS $=$ not significant.

comprising only $12 \%$ of eyes with neovascular glaucoma. ${ }^{12}$ Similarly, Spencer et al reported a low (3.4\%) incidence of hypotony in a series of 58 eyes, of which only six had neovascular glaucoma. ${ }^{13}$ Conversely, in a more recent study in which neovascular glaucoma accounted for $60 \%$ of the patients, the hypotony rate was $18 \%{ }^{15}$ However, other

Table 5 Distribution of eyes by number of cyclodiode treatment sessions

\begin{tabular}{lcc}
\hline $\begin{array}{l}\text { No of treatment } \\
\text { sessions }\end{array}$ & No of eyes & \% of eyes \\
\hline 1 & 173 & 65.8 \\
2 & 67 & 25.5 \\
3 & 13 & 4.9 \\
4 & 5 & 1.9 \\
5 & 1 & 0.4 \\
6 & 2 & 0.8 \\
7 & 2 & 0.8 \\
\hline
\end{tabular}




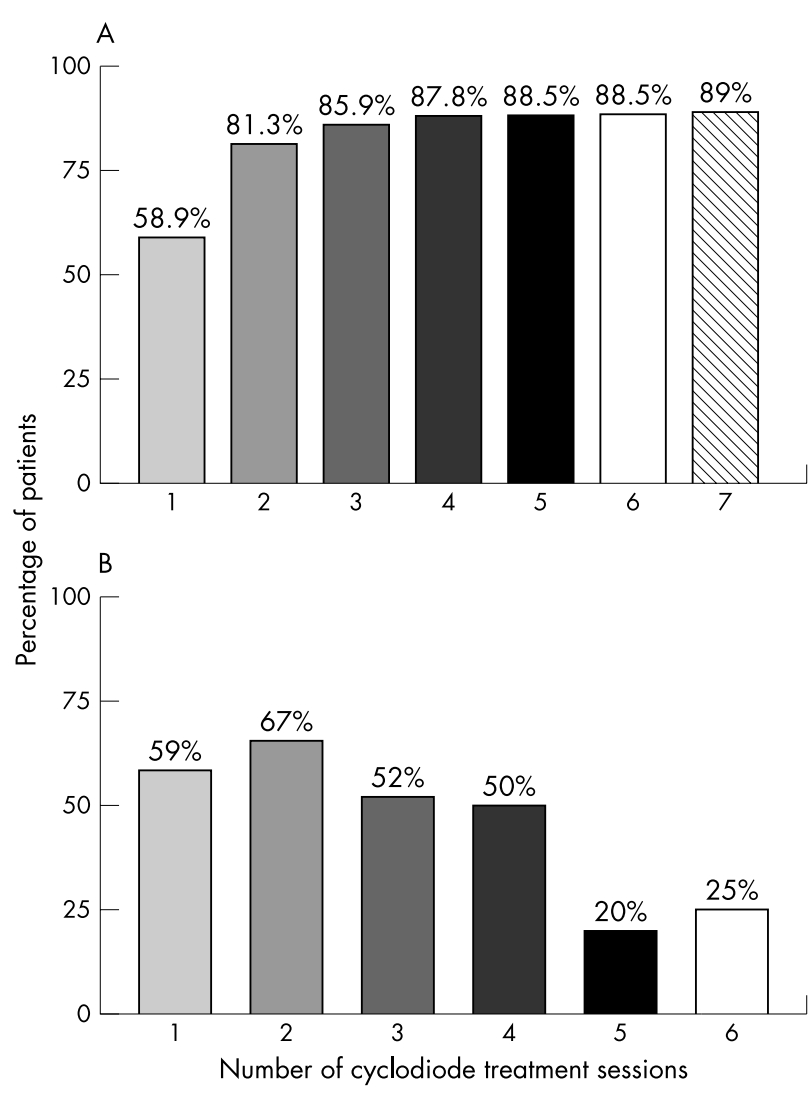

Figure 3 Response rate for cyclodiode therapy. (A) shows the percentage of patients achieving an adequate response (IOP $<22 \mathrm{~mm} \mathrm{Hg}$ or a drop in IOP $>30 \%$ ) following each additional treatment episode-that is, the cumulative response rate. (B) shows the response rate for each individual treatment episode.

variables might also influence the occurrence of hypotony, such as method of laser delivery, treatment settings, and follow up period. The mean energy per treatment session of 104.1 $\mathrm{J}$ in this series is higher than that reported by other authors. Spencer and Vernon and Bloom et al delivered mean energies of $56 \mathrm{~J}$ and $90 \mathrm{~J}$ in their studies, respectively, and this, together with their lower proportion of neovascular glaucoma cases, may explain their lower hypotony rates. ${ }^{12}{ }^{13}$ The relatively high incidence of hypotony in the uveitis group of the present study is also a cause for concern, and is at variance with a recent report of 18 patients with uveitic glaucoma in whom no serious adverse effects were reported. ${ }^{16}$

In the present study we investigated whether postcyclodiode hypotony could be predicted on the basis of age, sex, pretreatment IOP, total laser energy delivered, mean laser energy per treatment episode, and the number of treatment episodes. Univariate regression analysis revealed that high pretreatment IOP and high mean energy per treatment episode were the only factors significantly associated with hypotony. High pretreatment IOP may cause ciliary body ischaemia, which in turn may lead to ciliary body shutdown following cyclophotocoagulation. The severity of disease affecting these eyes was indicated by their precyclodiode visual acuity, which was hand movements or worse in all cases with a median value of perception of light. That high mean energy per treatment episode was associated with hypotony was not unexpected. In retrospect, a safer approach might have been to apply a maximum of 60-80 J of energy during each treatment session and have given multiple treatments at intervals of at least 6-8 weeks if the IOP response was poor.
Although transscleral diode laser cyclophotocoagulation has been established as a relatively safe and effective treatment for refractory glaucoma, no consensus exists on the optimum treatment protocol. Thus, while standard protocols have been evaluated, there is a lack of substantive randomised trials comparing the outcome of different protocols. ${ }^{4}{ }^{12} 13$ This study has investigated the relation between the total energy delivered during a course of cyclodiode therapy for refractory glaucoma and the reduction in IOP over a range of laser energies. In this series, which comprises a wide variety of glaucoma aetiologies, a linear dose-response relation did not exist that would help predict the outcome of cyclodiode therapy, a finding which is consistent with two previous reports on the IOP response in 30 and 33 eyes, respectively. ${ }^{15}{ }^{17}$ Interestingly, in neovascular glaucoma, the largest subgroup in our analysis comprising 122 eyes, the dose response appeared linear. However, since the risk of hypotony was high in neovascular glaucoma, especially in those with high prelaser IOP and those receiving high energy treatments, it is proposed that a more cautious approach with the initial delivery of a relatively low dose of diode laser energy might minimise the risk of hypotony in this group.

Although only $11 \%$ of eyes failed to achieve an intraocular pressure of 5-21 mm Hg or a 30\% drop in IOP following one or more treatment sessions, an attempt was made to identify factors that might help predict a poor IOP response. The analysis revealed that male sex was associated with treatment failure $(p=0.008)$, but an explanation for this difference was difficult to propose. It was reassuring to note that the response rate remained over $50 \%$ for each of the first four treatment sessions in patients who required multiple treatment sessions to gain IOP control, suggesting that it is worth persevering with treatment when the initial response is inadequate. However, subsequent treatments were associated with a much lower response rate in the region of 20 $25 \%$, indicating that the law of diminishing returns may apply when multiple $(>4)$ cyclodiode treatments are given. This information may be useful when counselling patients on the likely outcome of diode laser cyclophotocoagulation.

In summary, the present study has demonstrated that diode laser cyclophotocoagulation is an effective treatment for refractory glaucoma of all aetiologies. Hypotony is the main risk of treatment and might be limited by reducing the laser energy applied to $40-80 \mathrm{~J}$ for patients who have very high pretreatment IOP, especially if associated with neovascular or uveitic glaucoma. The dose response to cyclodiode therapy remains unpredictable, although a linear relation was found for eyes with neovascular glaucoma.

However, the optimum treatment parameters for cyclodiode therapy remain unclear and a prospective trial may be required to establish a safe and predictable treatment protocol. The high success rate and low risk of complications in some diagnostic groups, especially primary open angle glaucoma, suggest that primary diode laser cyclophotocoagulation may be a safe alternative to trabeculectomy in certain patients with POAG who have failed with medical therapy.

\section{ACKNOWLEDGEMENTS}

We thank Mr John Sparrow from the Bristol Eye Hospital for his statistical advice.

\section{Authors' affiliations}

C C Murphy, Division of Ophthalmology, University of Bristol, Bristol, UK C A M Burnett, D C Broadway, Department of Ophthalmology, Norfolk and Norwich University Hospital, Norwich, UK

P G D Spry, J P Diamond, Department of Ophthalmology, Bristol Eye Hospital, Bristol, UK 


\section{REFERENCES}

1 Hennis HL, Stewart WC. Semiconductor diode laser transscleral cyclophotocoagulation in patients with glaucoma. Am J Ophthalmol 1992;113:81-5.

2 Hawkins TA, Stewart WC. One-year results of semiconductor transscleral cyclophotocoagulation in patients with glaucoma. Arch Ophthalmol 1993;111:488-91

3 Kosoko O, Gaasterland DE, Pollack IP, et al. Long-term outcome of initial ciliary ablation with contact diode laser transscleral cyclophotocoagulation for severe glaucoma. The diode laser ciliary ablation study group. Ophthalmology 1996;103:1294-302.

4 Brancato R, Carassa RG, Bettin P, et al. Contact transscleral cyclophotocoagulation with diode laser in refractory glaucoma. Eur J Ophthalmol 1995:5:32-9.

5 Threlkeld AB, Johnson MH. Contact transscleral diode cyclophotocoagulation for refractory glaucoma. J Glaucoma 1999;8:3-7.

6 Egbert PR, Fiadoyor S, Budenz DL, et al. Diode laser transscleral cyclophotocoagulation as a primary surgical treatment for primary openangle glaucoma. Arch Ophthalmol 2001;119:345-50.

7 Mistlberger A, Liebmann JM, Tschiderer $\mathrm{H}$, et al. Diode laser transscleral cyclophotocoagulation for refractory glaucoma. J Glaucoma 2001; 10:288-93.

8 Benson MT, Nelson ME. Cyclocryotherapy: a review of cases over a 10-year period. Br J Ophthalmol 1990;74:103-5.
9 Schuman JS, Bellows AR, Shingleton BJ, et al. Contact transscleral Nd:YAG laser cyclophotocoagulation. Midterm results. Ophthalmology 1992;99:1089-94

10 Ulbig MW, McHugh DA, McNaught Al, et al. Clinical comparison of semiconductor diode versus neodymium:YAG non-contact cyclophotocoagulation. Br J Ophthalmol 1995;79:569-74.

11 Schlote T, Derse M, Rassmann K, et al. Efficacy and safety of contact transcleral diode laser cyclophotocoagulation for advanced glaucoma. J Glaucoma 2001;10:294-301.

12 Bloom PA, Tsai JC, Sharma K, et al. Cyclodiode: trans-scleral diode laser cyclophotocoagulation in the treatment of advanced refractory glaucoma. Ophthalmology 1997; 104:1508-19.

13 Spencer AF, Vernon SA. "Cyclodiode": results of a standard protocol. Br J Ophthalmol 1999:83:311-16.

14 Kirwan JF, Shah P, Khaw PT. Diode laser cyclophotocoagulation: role in the management of refractory pediatric glaucomas. Ophthalmology 2002;109:316-23

15 Walland MJ. Diode laser cyclophotocoagulation: longer term follow up of a standardized treatment protocol. Clin Exp Ophthalmol 2000;28: 263-7.

16 Schlote T, Derse M, Zierhut M. Transscleral diode laser cyclophotocoagulation for the treatment of refractory glaucoma secondary to inflammatory eye diseases. Br J Ophthalmol 2000;84:999-1003.

17 Wong EYM, Chew PTK, Chee CKL, et al. Diode laser contact transscleral cyclophotocoagulation for refractory glaucoma in Asian patients. Am 5 Ophthalmol 1997; 124:797-804.

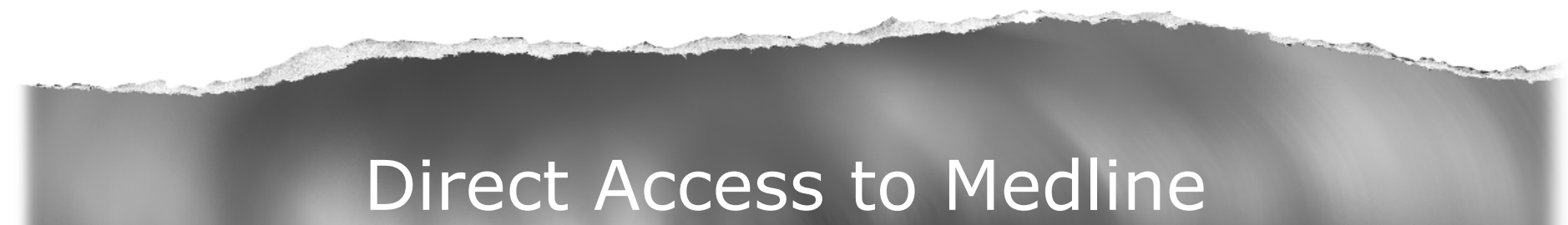

\section{Medline}

Link to Medline from the homepage and get straight into the National Library of Medicine's premier bibliographic database. Medline allows you to search across 9 million records of bibliographic citations and author abstracts from approximately 3,900 current biomedical journals.

\section{www.bjophthalmol.com}

